\title{
Decreased Firing of Striatal Neurons Related to Licking during Acquisition and Overtraining of a Licking Task
}

\author{
Chris C. Tang, David H. Root, Dawn C. Duke, Yun Zhu, Kate Teixeria, Sisi Ma, David J. Barker, and Mark 0. West \\ Department of Psychology, Rutgers University, New Brunswick, New Jersey 08903
}

Neurons that fire in relation to licking, in the ventral part of the dorsolateral striatum (DLS), were studied during acquisition and performance of a licking task in rats for 14 sessions $(2 \mathrm{~h} / \mathrm{d})$. Task learning was indicated by fewer errors of omission of licking and improved movement efficiency (i.e., shorter lick duration) over sessions. Number of licks did not change over sessions. Overtraining did not result in habit formation, as indicated by similar reductions of licking responses following devaluation by satiety in both early and late sessions. Twenty-nine lick neurons recorded and tracked over sessions exhibited a significant linear decrease in average firing rate across all neurons over sessions, correlating with concurrent declines in lick duration. Individually, most neurons (86\%) exhibited decreased firing rates, while a small proportion (14\%) exhibited increased firing rates, during lick movements that were matched over sessions. Reward manipulations did not alter firing patterns over sessions. Aside from the absence of habit formation, striatal processing during unconditioned movements (i.e., licking) was characterized by high activity of movement-related neurons during early performance and decreased activity of the same neurons during overtraining, similar to our previous report of head movement neurons during acquired, skilled, instrumental head movements that ultimately became habitual (Tang et al., 2007). Decreased activity in DLS neurons may reflect a common neural mechanism underlying improvement in movement efficiency with overtraining. Nonetheless, the decreased striatal firing in relation to a movement that did not become habitual demonstrates that not all DLS changes reflect habit formation.

\section{Introduction}

Neurons that fire phasically in relation to sensorimotor activity of single body parts (SBP neurons) (Cho and West, 1997) are useful for studying corticostriatal plasticity. Their distribution and somatotopic organization in the lateral, or sensorimotor, striatum (Liles, 1979; Crutcher and DeLong, 1984a,b; Alexander and DeLong, 1985; Liles and Updyke, 1985; Carelli and West, 1991; Mittler et al., 1994; Cho and West, 1997) correspond to topographic projections from motor and somatosensory cortices (Künzle, 1975, 1977; Selemon and Goldman-Rakic, 1985; McGeorge and Faull, 1989; Ebrahimi et al., 1992; Flaherty and Graybiel, 1993, 1994; Kincaid and Wilson, 1996), the convergence of which is reflected in SBP neuron firing (West et al., 1990; Carelli and West, 1991; Flaherty and Graybiel, 1993). SBP neurons are type II medium spiny projection neurons (Kimura et al., 1990), projecting via the pallidum and substantia nigra to motor thalamus and back to premotor cortical areas (Alexander et al., 1986; Parent and Hazrati, 1993; McFarland and Haber, 2000). SBP neurons are ideally situated for motor control and learning;

\footnotetext{
Received June 10, 2009; revised 0ct. 6, 2009; accepted 0ct. 10, 2009.

This study was supported by National Institute on Drug Abuse Grants DA 06886, DA 04551, and DA 026252 and National Institute of Mental Health Grant MH 0919957. We thank Linda King, Patrick Grace, Harry Ting, and Jaya Sandoghar for technical assistance.

Correspondence should be addressed to Dr. Mark 0. West, Department of Psychology, Rutgers University, 152 Frelinghuysen Road, Piscataway, NJ 08903. E-mail: markwest@rutgers.edu.

Dr. C. C. Tang's present address: Center for Neurosciences, The Feinstein Institute for Medical Research, North Shore-Long Island Jewish Health System, Manhasset, NY 11030.

Dr. D. C. Duke's present address: University of Surrey, Guildford, Surrey GU2 7XH, UK.

Y. Zhu's present address: Merck \& Co., Inc., One Merck Drive, Whitehouse Station, NJ 08889.

DOI:10.1523/JNEUROSCI.2824-09.2009

Copyright $\odot 2009$ Society for Neuroscience ～0270-6474/09/2913952-10\$15.00/0
}

compromised corticostriatal function yields motor deficits in Parkinson's patients (Marsden, 1982), including deficits in acquiring motor programs (Sheridan et al., 1987).

Habit learning involves corticostriatal processing (Mishkin and Petri, 1984). While the striatum appears necessary for acquisition of motor habits (Yin et al., 2004; Faure et al., 2005), it is unclear whether the striatum is necessary for their subsequent expression. Yin et al. (2004) found that pre- but not post-training lesions of the dorsolateral striatum (DLS), in which forelimb SBP neurons are located (Cho and West, 1997), resulted in reduced lever pressing for sucrose after outcome devaluation, suggesting that the DLS is necessary for habit acquisition but not expression. In contrast, Graybiel (2000) proposed that the basal ganglia are necessary for both stages of habit learning. A difficulty in interpreting deficits produced by post-training lesions, however, is that striatal damage causes abnormal activity in pallidal and nigral efferents to thalamus and premotor cortex (Filion et al., 1988; Tremblay et al., 1989; Rothblat and Schneider, 1995; Raz et al., 2001), which could interfere with expression of motor programs stored in premotor areas or disrupt sensorimotor processing such that familiarity with the habitual situation is lost.

To avoid this difficulty, we have recorded SBP neuronal activity during and after learning of skilled movements. Neurons related to forelimb movement fired in relation to lever pressing during early but not late sessions (Carelli et al., 1997). Neurons related to head movement changed firing rate (FR) (mostly decreases) as instrumental head movements became more efficient and habitual (Tang et al., 2007). It is, however, unknown whether changes in striatal firing will occur while repeatedly performing a consummatory movement that is unlikely to become habitual 
(Balleine et al., 1995). We therefore examined striatal firing during acquisition and extended training in a task requiring a familiar movement, i.e., licking. SBP neurons that fire in synchrony with individual licks, located within the ventral part of the DLS (Alexander and DeLong, 1985; Mittler et al., 1994; Cho and West, 1997), were examined.

\section{Materials and Methods Subjects and surgery}

Male (300-350 g) Long-Evans rats (Charles River) $(n=13 ; 11$ for neural recordings and 2 for devaluation only) were used as subjects. Before surgery, subjects received injections of atropine methyl nitrate $(10 \mathrm{mg} /$ $\mathrm{kg}$, i.p.) and penicillin $\mathrm{G}(75,000 \mathrm{U} / 0.25 \mathrm{ml}$, i.m.) to protect subjects from airway congestion and infection, respectively. Rats were anesthetized with sodium pentobarbital $(50 \mathrm{mg} / \mathrm{kg}$, i.p.). Anesthesia was maintained with periodic intraperitoneal injections of ketamine hydrochloride (60 $\mathrm{mg} / \mathrm{kg}$, i.m.). An array of Teflon-coated, stainless steel microwires (Shaptek Services) was implanted into the ventral part of the right, dorsolateral striatum (between -0.6 and $1.2 \mathrm{~mm}$ AP; between 3.2 and $4.2 \mathrm{ML}$; and 6.0 DV from skull) (Paxinos and Watson, 2005) and secured with dental cement. The array consisted of 12 microwires (diameter of each uninsulated tip, $50 \mu \mathrm{m}$ ), arranged in two parallel rows, which were $\sim 2 \mathrm{~mm}$ in length and separated from one another by $0.45-0.55 \mathrm{~mm}$ (wire center to wire center). An insulated 0.01 inch wire, stripped $5 \mathrm{~mm}$ from the tip, was implanted $7 \mathrm{~mm}$ ventral from skull level on the left side of the brain as a ground to minimize noise during electrophysiological recording. Antibiotic was applied to the incision and sutures after completion of the surgery.

After surgery, rats were individually housed with access to food and water to recover for at least 1 week. The colony room was maintained on a reversed, $12 \mathrm{~h} / 12 \mathrm{~h}$ light/dark cycle (on 20.00, off 8.00) so that recording/training sessions occurred during the rats' active portion of the cycle. When rats reached a body weight of 380-390 g during recovery, water deprivation was started so that body weights gradually dropped to $\sim 82 \%$ of their maximal ad libitum feeding weight at 311-319 g and were maintained at this for the entire duration of the experiment.

\section{Equipment}

A water spout was positioned outside the front wall of a transparent Plexiglas recording chamber $(23.5 \times 17.4 \times 43 \mathrm{~cm})$. Silicon tubing was used to connect a container filled with tap water, and a solenoid to a stainless steel drinking spout located $2 \mathrm{~mm}$ from the chamber. There was a small hole in the Plexiglas ( $7 \mathrm{~mm}$ diameter) to allow the rat's tongue access to water drops. A photo lick sensor, consisting of fiber optic below and above the spout, was used to register individual licks. The presence or absence (during a lick) of the light beam was recorded and time stamped every $16.6 \mathrm{~ms}$ (Datawave Technologies), simultaneously with the time stamping of neural waveforms $(0.1 \mathrm{~ms})$. Water delivery was noncontingent on behavior, controlled by a transistor-transistor logic pulse from the computer that opened the solenoid for $35 \mathrm{~ms}$, delivering one drop of $\sim 5 \mu \mathrm{l}$ through the spout (one water delivery). Time between water deliveries was pseudorandom, ranging from 6 to $12 \mathrm{~s}$, mean $=9 \mathrm{~s}$. An audible tone $(3 \mathrm{kHz})$ was sounded through a speaker mounted above the chamber for the duration that the solenoid was open to make the activation of the solenoid audible to the animal. Water delivery, licks, and neural firing were processed and stored by a microcomputer. White noise was used to prevent any disturbances from outside the experimental room.

\section{Experimental design}

\section{Licking task}

Rats were trained in a licking task for 14 consecutive sessions ( $2 \mathrm{~h}$ per session, 1 session per day). Before the day of the first session, a preliminary exam was conducted to determine which wires were recording lick neurons. Animals were allowed $10 \mathrm{~min}$ to acclimate to the chamber before examination of neural activity. During the exam, drops of water were manually delivered on the floor to determine neural FR during licking. A complete sensorimotor exam was also performed to determine whether neural firing was correlated with sensorimotor stimulation of any body part (Cho and West, 1997; Pederson et al., 1997). Only neurons that (1) unconditionally increased FR during licking and (2) did not increase FR correlated with any nonoral behavior were recorded during an experiment.

Following the preliminary exam, recording sessions in the task commenced. Each rat was placed in the chamber and a recording harness was attached to the microwire array. The session lasted $2 \mathrm{~h}$ (one session per day), which consisted of six cycles: six water-on phases (15 min) alternating with six water-off phases (5 min).

During the $2 \mathrm{~h}$ session, the rat received $\sim 3 \mathrm{ml}$ of water. After each session, the rat was given another $10 \mathrm{ml}$ of water to reach the daily amount of water $(\sim 13 \mathrm{ml})$ needed to maintain stable weight.

\section{Devaluation test}

Six rats underwent a devaluation procedure in two sessions (sessions 2 and 13) to determine whether a licking habit was formed after repeated training sessions, as defined by Dickinson (1985). The rat was given its daily allotment of water $(13 \mathrm{ml})$ immediately before the devaluation session (food was available but all rats were observed consuming only water) and then immediately placed in the task with all other experimental conditions unchanged.

\section{Neural recording}

During an experiment, neural signals were led through a preamplifier that differentially amplified the signal on the recording electrode against another microwire within the striatum that did not exhibit a neural signal. The signal was then led through a bandpass filter $(450 \mathrm{~Hz}$ to $10 \mathrm{kHz}$; roll off $1.5 \mathrm{~dB}$ /octave at $1 \mathrm{kHz}$ and $-6 \mathrm{~dB} /$ octave at $11 \mathrm{kHz}$ ). Using software and hardware of DataWave Technologies, electrical signals were sampled (62.5 kHz sampling frequency for each wire) and stored for off-line analysis.

\section{Change of experimental conditions in session 15}

In session $15,0.15 \%$ saccharin solution or $32 \%$ sucrose replaced water as a reward. All other experimental conditions remained unchanged.

\section{Histology}

Using histological procedures described previously (Tang et al., 2008), the location of each recorded neuron was determined by reconstructing its three-dimensional position within the striatum according to the atlas of Paxinos and Watson (2005). Neurons recorded by wire tips localized outside the ventral part of the DLS were discarded.

\section{Data analysis}

\section{Behavioral data analysis}

Onset and end time of licks. The onset of each lick was defined as the time at which the fiber optic light beam was blocked. The lick end time was defined as the time at which the light beam became unblocked.

Lickduration, period, reaction time, total licks, water-on licks, and wateroff licks. Lick duration was the time between lick onset and end time. Period was the time between onset of the present lick and onset of the next lick. Reaction time was defined as the delay from the delivery of the first water drop beginning each water-on phase to the onset of the first lick in that phase. The average reaction time of all six water-on phases was calculated for each session for each rat. Total licks were all licks made by each rat during an entire $2 \mathrm{~h}$ session. Water-on licks were all licks in six 15 min water-on phases in each session for each rat. Water-off licks were all licks in six 5 min water-off phases in each session for each rat.

To assess acquisition of the task, reaction time, total licks, and water-on licks were each analyzed by using a one-way repeated-measures ANOVA (RMANOVA) across all rats over all 14 sessions, followed by post hoc Scheffe's tests between each of the subsequent sessions and session 1 with adjustment for multiple comparisons. The data from devaluation sessions were excluded from this analysis but were analyzed separately to evaluate formation of a licking habit.

To assess habit formation, changes in total licks, water-on licks, and water-off licks were assessed in devalued and nondevalued rats, separately, across all sessions. Differences in the number of licks were also 
compared between early and late devaluation sessions using nonparametric within-subject sign tests. In addition, a $2 \times 2$ RMANOVA was used to compare changes in water-on and water-off licks between early and late devaluation sessions for devalued rats. To control for individual differences in task performance of devalued rats during water-on and water-off phases, this analysis was performed on the ratio of the number of licks in each devaluation session to the number of licks in the immediately preceding session for each rat.

The duration and period of each lick were used to generate "matched sets" (see below) that were used for both behavioral and neural analysis. Changes in these movement parameters were assessed over sessions. Period was divided into 12 equal increments from low to high: 84 to $267 \mathrm{~ms}$. Median duration of each period level was calculated for licks in each session of each rat. One-way RMANOVA was used to assess changes in lick duration over sessions, controlling for period levels by including this parameter as a covariate. Similarly, lick duration was divided into seven equal levels from low to high: 17 to $117 \mathrm{~ms}$. The median period of each duration level was calculated for each session for each rat. One-way RMANOVA was also used to assess changes in period over sessions, controlling for duration levels by including it as a covariate.

\section{Neural data analysis}

Determining waveforms of single neurons in every session. The isolation and separation of the waveforms of each individual neuron were conducted post hoc using the "cluster-cutting" process of the Datawave analysis software (Personal Scientific Workstation and Discovery Acquisition, Datawave Technologies) as described previously (Tang et al., 2008). All waveforms of the putative individual neuron during the entire session $(2 \mathrm{~h})$ were replayed on a computer-simulated oscilloscope to assess the stability of neural waveforms within a session. Waveforms whose parameters did not remain stable within a session were discarded. Then, an interspike interval (ISI) histogram was constructed to confirm that the isolated waveforms were from single neurons. If discharges occurred within the first $2 \mathrm{~ms}$ in the ISI histogram, corresponding to a neuron's natural refractory period, the recording was not considered that of a single neuron and therefore was discarded.

Identifying the same single neuron recorded over sessions. Similar to our previous investigation (Tang et al., 2007), a key assumption of the present study was that the stability in recordings observed day to day allowed the activity of the same single neuron to be recorded on-line and tracked off-line over sessions. To establish that the same single neuron was recorded over sessions, the following criteria had to be met: (1) Present throughout the time in question (minimum 4 sessions; maximum 14 sessions) and recorded from the same microwire; (2) Similar waveforms and ISI histograms throughout recordings. Neurons whose waveform parameters and/or ISI histograms showed apparent changes throughout the time studied were either discarded or used only until the time of loss in stability (minimum of four sessions); (3) Absence of discharges within the first $2 \mathrm{~ms}$ of the ISI histogram, consistent with the natural refractory period of a single neuron (Kosobud et al., 1994; Peoples et al., 1998); (4) If one wire exhibited two different waveforms meeting the above criteria (not common), a cross-correlogram was constructed. If spikes occurred within the interspike interval $(2 \mathrm{~ms})$ that reflected the natural refractory period of a single neuron, then it was concluded that the two waveforms, each with its own refractory period, represented different neurons.

Construction of perievent time histograms. Preliminary to neural analysis, a perievent time histogram (PETH) that displayed neural firing within -1000 to $+1000 \mathrm{~ms}$ around the onset of each lick was constructed for each neuron. A neuron's firing was determined to be correlated with licking if and only if firing increased visibly around the time of the lick. Only neurons that showed a correlation with licking both in the sensorimotor exam before the experiment and in the PETH were finally confirmed as lick neurons and entered into the subsequent neural analysis. Some neurons increased FR before lick onset, some at the onset, and some after onset. Therefore, analysis of neural firing was customized to the individual neuron. A neuron's FR associated with each lick (see below, Matched sets) was calculated by dividing the number of discharges that occurred in the firing window by the duration of that firing window. The vast majority of licks were associated with either one or no discharge.

Matched sets. Previous studies in this laboratory demonstrated that two parameters (duration and preceding period) may show significant correlations with firing of lick neurons (Tang et al., 2005, 2008). Therefore, licks were analyzed according to these two parameters. In a recent study, we have applied the method of matching licks between two consecutive experimental conditions (saline vs cocaine) in analyzing changes in firing rates of lick neurons (cf. Materials and Methods, Tang et al., 2008). In the present study, this method was extended to generate matched sets of similar licks across up to 14 sessions.

For each lick, the firing rate and the values of the two behavioral parameters for that lick were entered into a SAS program. For any given session, licks were selected post hoc into matched sets stochastically, without regard to time of occurrence or phase (water-on or -off). In the program, licks during each session were sorted on the basis of each lick's duration and period. Then, duration was divided into 7 equal increments from low to high: 17 to $117 \mathrm{~ms}$; and period was divided into 12 equal increments from low to high: 84 to $267 \mathrm{~ms}$. Thus, the format of the SAS output file was a two-dimensional spreadsheet containing 84 cells (duration $\times$ period). Each cell tallied a unique set of movements whose parameters were similar to each other but different from those in any other cell. Out of this format, two spreadsheets having 84 cells with the same values of duration and period were made for each session for every neuron. One contained the number of observed movements $(n)$ in each cell; the other contained the mean firing rate of all observed movements in each cell, calculated by dividing the sum of the firing rates of individual movements by the number of movements in each cell. Many cells contained no movements because certain types of movements were not possible or did not occur (e.g., very long duration with very short period). In a given session, only cells with at least five movements $(n \geq 5)$ were included into further analyses with respect to firing. Cells with the same movement parameters in spreadsheets generated in different sessions for the same single neuron, along with their corresponding mean firing rates, were then matched across sessions, yielding matched sets.

Thus, a matched set was defined as the set of licks with the same behavioral parameters in corresponding cells of the spreadsheets observed in different sessions throughout training. All assessments of changes in firing across sessions were based on comparing the mean firing rates associated with movements of an individual matched set. In other words, all comparisons of firing were among movements that were precisely matched to movements in other sessions. Therefore, the analysis of matched sets enabled analysis of changes in firing of the same single neuron across training sessions while holding movement parameters "constant."

Modeling the firing rates of matched sets with a three-level individual model. The neural data had a three-level hierarchical structure: withinmatched set/within-neuron/between-neuron. Specifically, a number of matched sets (level 1) were nested within individual neurons (level 2), which were nested within (or belong to) a population of lick neurons (level 3). To analyze these neural data, a corresponding three-level individual growth model was used to examine the average change in firing across all matched sets of all neurons over sessions. Both linear (i.e., session) and curvilinear (i.e., session ${ }^{2}$ ) changes were modeled to determine the trajectory of the average FR over sessions. Lick parameters (i.e., duration and period) were also added into the model to examine the influence of these parameters on changes in FR. In the stepwise modeling, improved fitting of the data was determined by comparing values of both the Akaike information criterion (AIC) and Bayesian information criterion (BIC) between models, i.e., the smaller the values, the better the model (Singer and Willett, 2003).

To further examine changes in firing of individual neurons, we fit a two-level individual growth model into the matched sets of each neuron. The resulting trajectories of changes in firing for individual neurons were also plotted. The sign of the slope was used to determine whether a neuron exhibited increased (positive slope) or decreased (negative slope) FR over sessions. The statistical characteristics of the individual growth model and its application to the analysis of multilevel neural data have been described in detail by us previously (Tang et al., 2007). In addition, 


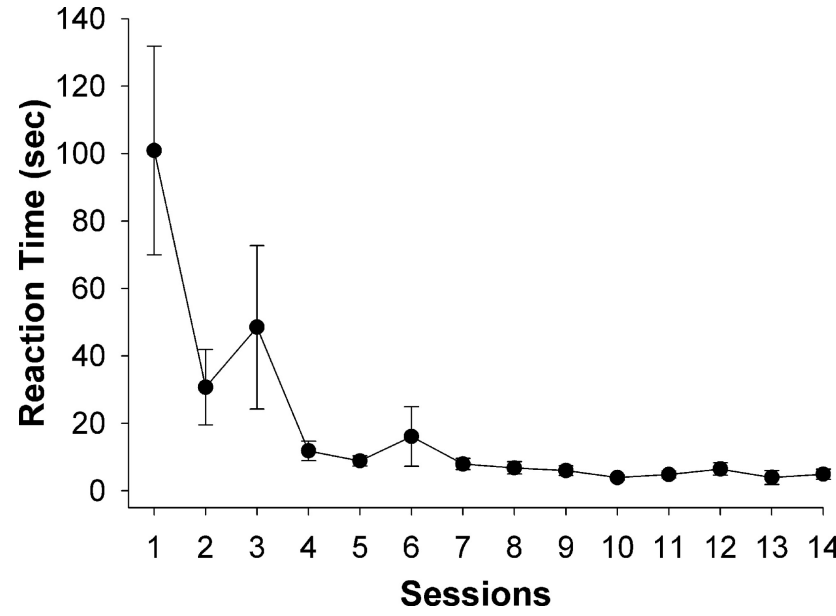

Figure 1. Decreased mean reaction time across sessions in the lick task $(p<0.001)$. Rats $(n=13)$ exhibited shorter reaction time in each of the subsequent sessions relative to session $1(p<0.02)$. Error bars represent SEM.

for lick parameters (duration and period) that were found to change significantly over sessions, the correlations of firing rates with the parameter(s) were calculated across all matched sets for each neuron in the first and last sessions, separately, in which it was recorded using nonparametric Spearman correlation coefficients $\left(r_{\mathrm{s}}\right)$. The correlations were then compared between the first and last sessions using Wilcoxon's signedranks test.

Analysis of changes in firing rate between sessions 14 and 15. Seven rats that exhibited lick neurons in session 14 were trained for one additional session (session 15) on the 15th day. In session 15, one change in experimental conditions was made: $0.15 \%$ saccharin solution (three rats) or $32 \%$ sucrose (four rats) was used instead of water as a reward, to assess the effect of this change on behavior and neural firing while all other experimental conditions were kept constant. For behavioral analysis, reaction time, total licks, water-on licks, and water-off licks were separately compared within rats between sessions 14 and 15 using nonparametric sign tests. For neural analysis, firing rates during similar lick movements between sessions 14 and 15 (termed "matched pairs") were generated for each neuron recorded in both sessions. Median firing rates of each neuron's matched pairs were calculated for both sessions. These median firing rates were then compared between sessions 14 and 15 for all neurons using a nonparametric sign test.

\section{Results}

Behavioral results

Thirteen rats were trained in the licking task over 14 sessions. Acquisition of the task was demonstrated by a significant decrease in reaction time over sessions $\left(F_{(13,135)}=5.67, p<0.001\right.$; RMANOVA). Relative to session 1, rats exhibited shorter reaction time in all subsequent sessions, reaching a stable performance between sessions 4 and 14 (Fig. 1) ( $p<0.02$; post hoc Scheffé's tests). Decreased reaction time in response to the auditory cue was not due to persistence of licking during the water-off phases because rats dramatically decreased the number of licks emitted within $1 \mathrm{~min}$ after the start of each water-off phase (data not shown).

Lick duration decreased significantly over sessions $\left(F_{(13,147)}=\right.$ $13.59, p<0.0001$; RMANOVA). Relative to session 1, rats exhibited shorter lick duration in each of the subsequent sessions (Fig. $2 A)$ ( $p<0.004$; post hoc Scheffé's tests). In contrast, although rats exhibited significant changes in lick period over sessions $\left(F_{(13,147)}=77.03, p<0.0001 ;\right.$ RMANOVA $)$, the changes were inconsistent, either increasing (sessions 2-7, 14: $p<0.05$ ), decreasing (session 9: $p<0.05$ ), or not changing (other sessions:
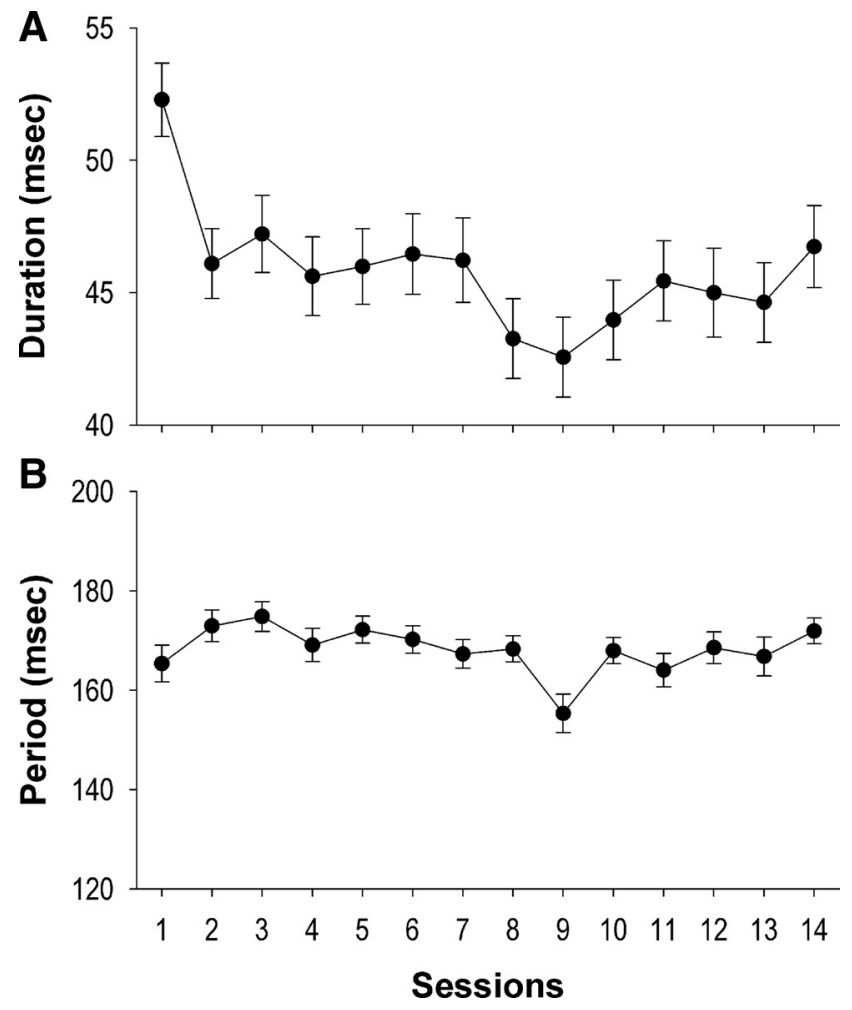

Figure 2. Changes in lick parameters across sessions in the lick task. $\boldsymbol{A}$, Lick duration significantly decreased over sessions ( $p<0.0001)$. Rats $(n=13)$ exhibited shorter lick duration in each of the subsequent sessions relative to session 1 ( $p<0.004)$. $\boldsymbol{B}$, Significant changes in lick period were also found over sessions $(p<0.0001)$. However, the changes were not consistent at the subsequent sessions, with increases (sessions 2-7, 14: $p<0.05$ ), decreases (session 9: $p<0.05$ ), or no changes (other sessions: $p>0.06$ ) relative to session 1. Error bars represent SEM.

$p>0.06$ ) relative to session 1 (Fig. 2 B) (post hoc Scheffé's tests). Moreover, rats did not show significant changes in the number of total licks ( $p=0.39$; RMANOVA) or the number of licks during water-on phases ( $p=0.48$; RMANOVA) over sessions.

To evaluate the formation of a licking habit, six rats underwent reward devaluation tests in sessions 2 and 13. We separately analyzed the numbers of licks over sessions in the devaluation $(n=6)$ and nondevaluation $(n=7)$ subgroups. In the nondevaluation subgroup, there were no changes in either total licks $\left(F_{(13,69)}=1.0, p=0.47 ;\right.$ RMANOVA $)$ or water-on licks $\left(F_{(13,69)}=0.76\right.$, $p=0.70$; RMANOVA) over sessions. In contrast, the devaluation subgroup exhibited significant changes in both measures over sessions (total licks: $F_{(13,65)}=7.82, p<0.001$; water-on licks: $F_{(13,65)}=7.43, p<0.001 ;$ RMANOVAs). Nevertheless, for both measures, devalued rats made significantly fewer licks only in the early (Fig. 3A) ( $p<0.005$; post hoc Scheffé's tests) and late $(p<$ $0.001)$ devaluation sessions relative to session 1; total licks and water-on licks did not differ between the early and late devaluation sessions ( $p>0.21$; nonparametric sign test). Together, these results demonstrate that licking was similarly reduced by the devaluation procedure both early and late in training, indicating that licking remained goal directed and that a habit was not formed after overtraining on the task.

Licking during water-off phases comprised both residual licking after the last water delivery of each water-on phase, as well as sporadic licking throughout the 5 min water-off phases. Across sessions, licking during water-off phases diminished but did not extinguish. Given that licking during water-off phases (1) per- 
A

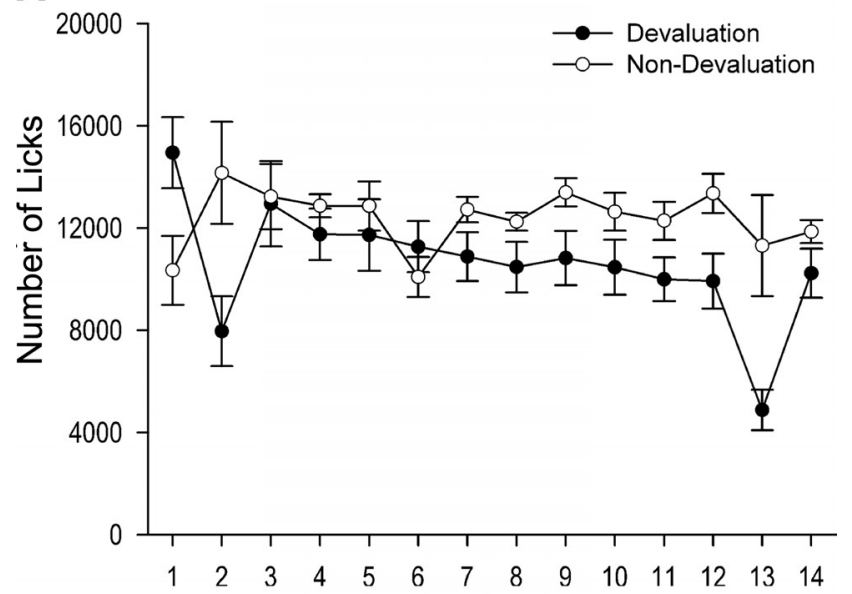

B

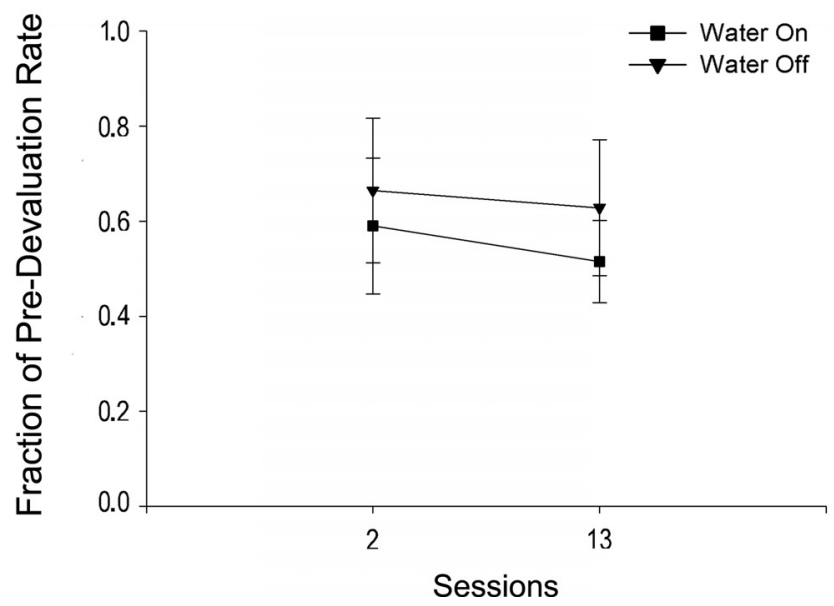

Figure 3. Lack of habit formation after repeated sessions in the lick task. $A$, Six rats (filled circles) underwent devaluation tests in sessions 2 and 13. These rats made significantly fewer total licks ( $y$ axis) in both the early $(p<0.005)$ and late $(p<0.001)$ devaluation sessions relative to session 1 , but total licks did not differ between the two devaluation sessions $(p>$ 0.21 ). The other, nondevalued rats ( $n=7$, open circles) did not show changes in total licks ( $p=0.47$ ) over sessions. $\boldsymbol{B}$, For the devalued rats, the normalized measure of licking, represented by the ratio of the number of licks in the devaluation session to the number of licks in the immediately preceding session ( $y$ axis), did not change between the early and late devaluation sessions ( $p=0.66$ ) in either the water-on (filled squares) or water-off (filled triangles) phases after overtraining ( $p=0.88 ; 2 \times 2$ RMANOVA). The fractions of predevaluation rates were similar between the early and late devaluation sessions $(p=0.66)$ in both the water-on and water-off phases for these rats. Error bars represent SEM.

sisted, (2) could (by definition) be nonconsummatory, and (3) occurred in the absence of the tone/solenoid, we tested whether it was sensitive to devaluation. Habit formation was tested by comparing differences in licking between the early and late devaluation sessions, during water-off versus water-on phases for devalued rats. Because of the greater number of licks in water-on versus water-off phases, and to control for individual differences, this analysis was based on a normalized measure for each rat, i.e., the ratio of the number of licks in the devaluation session to the number of licks in the immediately preceding session. There was no significant devaluation session $X$ water-on/off phase interaction effect (Fig. $3 B)\left(F_{(1,10)}=0.25\right.$, $p=0.88 ; 2 \times 2$ RMANOVA) nor main effects of devaluation session $(p=0.66)$ or water-on/off phase $(p=0.52)$. Thus, habit formation was not evident in either water-on or wateroff phases after overtraining.

\section{Neural results}

Twenty-nine lick neurons were recorded over sessions. Of these neurons, 21 (72\%) were each recorded from a single microwire; for the remaining 8 neurons, four pairs of 2 neurons were recorded from individual microwires. All neurons were histologically verified to be in the ventral part of the DLS (Fig. 4). Of all 29 neurons, 19 (66\%) exhibited neural activity recorded throughout all 14 training sessions; the remaining 10 neurons were recorded for a range of 7-12 sessions. The mean stable recording for all neurons was 12.2 ( \pm 0.5 ; SEM) sessions. A total of 1793 matched sets were obtained from these neurons with an average of 62 $( \pm 15$; SD) matched sets for each neuron. A three-level individual growth model on the neural data revealed a significant linear decrease in firing rate (slope $=-0.34 \mathrm{impulses} / \mathrm{s} / \mathrm{session}, p<$ 0.005 ) across all matched sets of all neurons over sessions (Table 1 , top). Adding lick duration into the model resulted in an improvement in fitting the data (i.e., smaller AIC and BIC values), with significant main effects of session $(p<0.001)$ and lick duration $(p<0.0001)$, and a significant interaction effect of lick duration $\times$ session $(p<0.0001)$ (Table 1 , bottom). Adding lick period or a curvilinear term (i.e., session ${ }^{2}$ ) into the model, however, did not further improve fitting of the data. Thus, the neural analysis suggested a linear decrease in the average FR of lick neurons associated with decreased lick duration over sessions (Fig. 5, bottom line). Note that the large decrease in firing, although correlated with the relatively small decrease in lick duration (Fig. $2 A$ ), was in no way a trivial result of reduced duration because of our use of matched sets to assess all changes in FR. As additional evidence of this, throughout sessions 2-14, the average lick duration maintained between 42 and $48 \mathrm{~ms}$ while firing during those licks decreased substantially.

After the linearity of the change in average FR across all neurons over sessions was determined, the trajectories of changes in firing for individual neurons were further examined by fitting a linear, two-level individual growth model into the matched sets of each neuron. Of the 29 neurons, 25 (86.2\%) showed decreased firing over sessions (Fig. 6, left, Fig. 7, example neuron). In these neurons, the rate of decrease (or slope) in FR correlated negatively $(r=-0.76 ; p<0.001)$ with the initial average firing rates, indicating that neurons with higher initial FR showed more rapid decreases in firing over sessions (Fig. 8). In addition, of the 25 neurons, 15 (60\%) exhibited a moderate or high correlation (i.e., $r_{\mathrm{s}}>0.3$ ) of firing with lick duration on the first session when the neuron was recorded, while $10(40 \%)$ exhibited a moderate or high correlation on the last recording session. For these neurons, there was a trend of decreased correlation between firing and lick duration ( $p=0.10$, Wilcoxon signed-ranks test) from the first (median $\left.r_{\mathrm{s}}=0.40\right)$ to last (median $r_{\mathrm{s}}=0.20$ ) sessions. Nonetheless, across neurons, the correlations between FR and lick duration in the first session were not associated with the initial average firing rates $(p=0.61)$ or with the rate of decrease in FR over sessions $(p=0.72)$.

In contrast, the remaining four neurons $(13.8 \%)$ showed increased firing over sessions (Fig. 6, right). Of these neurons, three (75\%; median $\left.r_{\mathrm{s}}=0.51\right)$ exhibited a moderate or high correlation of firing with lick duration on the first recording session and three $\left(75 \%\right.$; median $\left.r_{\mathrm{s}}=0.60\right)$ exhibited a moderate or high correlation on the last recording session, suggesting that the correlation was maintained for these neurons over sessions.

Eighteen neurons from seven rats were recorded in both sessions 14 and 15. For these rats, none of the behavioral measures (reaction time, total number of licks, the numbers of water-on and water-off licks) changed between sessions 14 and 15 ( $p>$ 


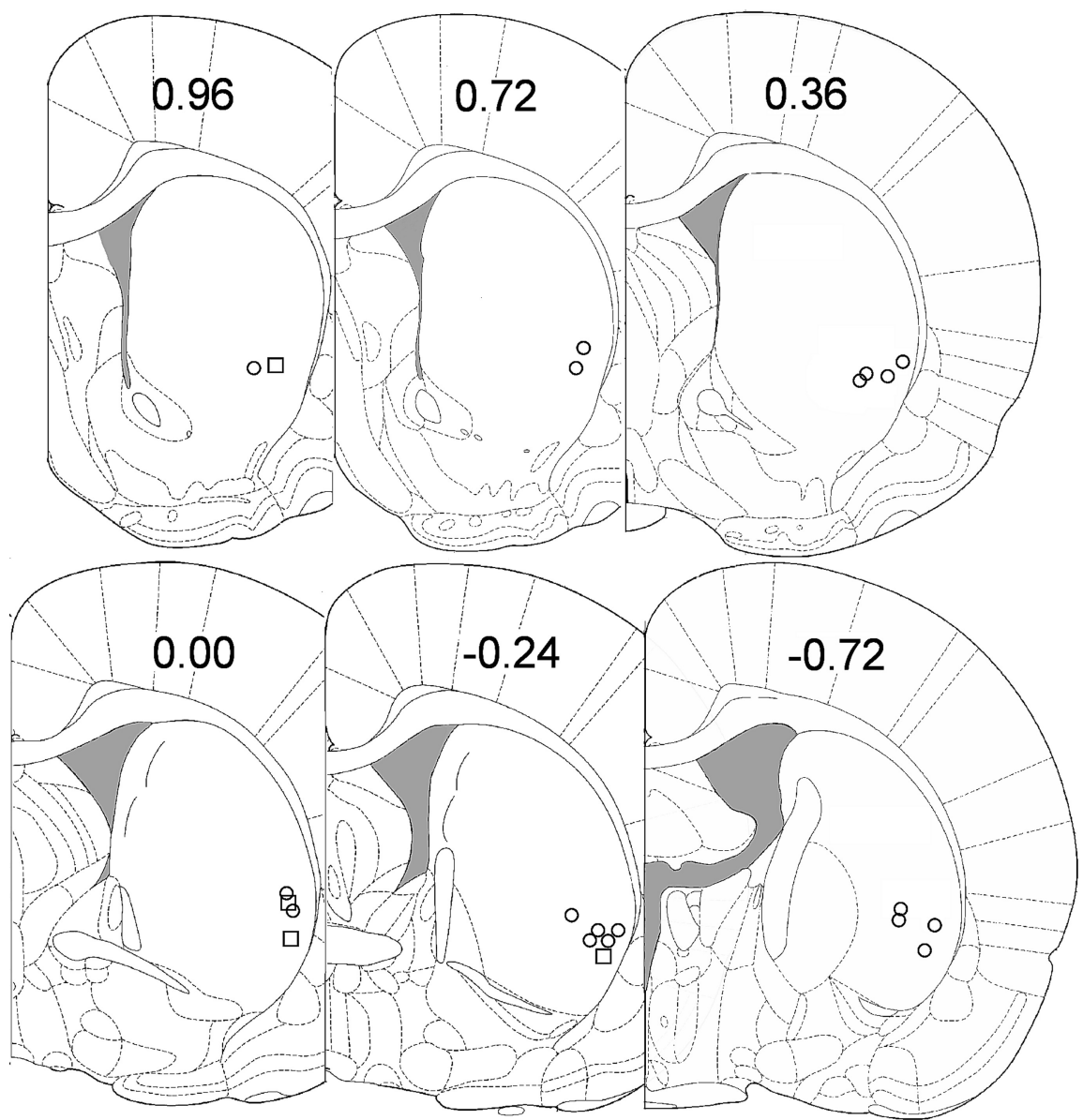

Figure 4. Coronal diagrams of the rat brain (Paxinos and Watson, 2005) showing the histologically verified microwire lesions in the ventral part of the dorsolateral striatum from which recordings of all 29 neurons were made. Numbers indicate anteroposterior distance from bregma (in millimeters).

Table 1. Results of three-level individual growth models on the neural data of matched sets

\begin{tabular}{lcrr}
\hline & Estimate \pm SE & $t$ value & $p$ value \\
\hline Model with session & & & \\
$\quad$ Intercept & $5.71 \pm 1.37$ & 4.15 & 0.0003 \\
$\quad$ Session & $-0.34 \pm 0.11$ & -3.05 & 0.0023 \\
Model with session and lick duration & & & \\
$\quad$ Intercept & $6.15 \pm 1.38$ & 4.47 & 0.0001 \\
$\quad$ Session & $-0.38 \pm 0.11$ & -3.43 & 0.0006 \\
$\quad$ Lick duration & $-0.008 \pm 0.002$ & -5.09 & $<0.0001$ \\
$\quad$ Lick duration $\times$ session & $0.0008 \pm 0.0002$ & 4.37 & $<0.0001$ \\
\hline
\end{tabular}

0.99; nonparametric sign tests). There were 530 matched pairs between sessions 14 and 15 obtained from the 18 neurons. There was no significant difference in firing rates of these matched pairs between the two sessions for these neurons ( $p=0.33$; nonparametric sign test). These results suggest that the change in reward using $0.15 \%$ saccharin solution or $32 \%$ sucrose to replace water in session 15 did not cause significant changes in either behavior or firing.

\section{Discussion}

The present findings demonstrate that the majority (86\%) of dorsolateral striatal neurons related specifically to licking exhibited a decrease in FR during licking as rats improved performance in the task with extended training sessions. Individual striatal neurons were demonstrated to fire significantly less than they had in early sessions during performance of the same licking movement, which presumably involved similar corticostriatal input from somatic sensorimotor cortices. With extensive practice, parameters of movement may be set and controlled through formation of a central motor program (Keele, 1968; Cooke, 1980; Meyer et al., 1988). Given that cortical representations typically increase with training (Nudo et al., 1996; Kleim et al., 1998), our data suggest that after acquisition of the licking task, the majority of striatal neurons may be less engaged by corticostriatal inputs and less involved in expression of skilled movements. This is consistent with the idea that the striatum may act to "train" the cortex in acquiring the motor program but contribute less as movements become automatic (Marsden, 1982; Wise et al., 1996; Pasupathy and Miller, 2005). Further, since $50-70 \%$ of DLS neurons are SBP neurons (Cho and West, 1997) and nonSBP DLS neurons also exhibit robust decreasing FRs during the same movements over days of training (Tang et al., 2007), decreasing FRs may be the primary pattern of change in DLS neurons across task and motor learning.

In contrast, a small percentage of striatal lick neurons (14\%) increased FR over repeated training sessions. These neurons continued to function in association with movement, possibly involved in maintenance of long-term storage of skilled movement. What factors might explain the difference between neurons exhibiting increasing versus decreasing FR? They are both type II medium spiny projection neurons (Kimura et al., 1990). In two cases, two neurons, one exhibiting an increasing pattern and the other a decreasing pattern over sessions, were recorded from the same microwire. The difference between increasing and decreasing patterns may involve different changes in responsiveness to input from cortex, one possibly involving long-term depression (Lovinger et al., 1993) and the other, long-term potentiation (Calabresi et al., 1997; Wickens et al., 1998). Alternatively, increasing or decreasing patterns could shape similar (Blomeley et al., 2009) or opposite patterns via connections among neighboring striatal neurons.

In previous studies, DLS forelimb neurons fired during early but not late sessions as rats acquired skill in a force lever task (Carelli et al., 1997), and the majority of DLS head movement neurons $(89 \%)$ exhibited a continuous decrease in FR as rats became skilled and habitual in a head movement task (Tang et al., 2007). Together with the present findings, the decreasing firing pattern in the majority has been consistently shown by different striatal neural populations in various motor learning paradigms. Decreased firing was also found to be associated with improved movement efficiency in all three studies. These data suggest that the DLS as a whole exhibits greater activity during task acquisition than during skillful performance of the task, and that the 
reduced striatal firing is associated with improved motor and/or task efficiency over extended training.

However, an important difference between these studies is that habitual behavior was not evident in the present study but was demonstrated in our previous examination of head movement neurons during an operant head movement task. In contrast to the head movement paradigm (Tang et al., 2007), the present experiment was designed so that the motor response as well as the environmental contingencies under which licking behavior occurred was inconsistent with the development of stimulus-response habitual responding. Behaviors induced by first-order appetitive conditioned stimuli, as used in the present experiment, are highly sensitive to the current value of the reward (Holland and Rescorla, 1975; Holland, 1979; Holland and Straub, 1979; Kerfoot et al., 2007). This was confirmed in the present study in which reward devaluation decreased the number of licks to a similar degree both early and late in training. Interestingly, magazine entries can remain goal-directed actions even though lever presses by the same animals can be resistant to reward devaluation, i.e., habitual (Balleine, 1992). This has led to the notion that actions proximal to reward consumption, such as licking, are persistently goal directed, while more distal behaviors, such as an operant head movement (Tang et al., 2007) or forelimb lever press (Carelli et al., 1997), are more likely to become habitual with overtraining (Balleine et al., 1995; Galarce et al., 2007; but see Faure et al., 2005).

Absence of habitual licking during water-off phases was also demonstrated. Licking during water-off phases amounted to $15-25 \%$ of the number of licks during water-on phases, indicating that animals discriminated between the two. But licking during water-off phases (1) persisted throughout all six phases of all sessions, (2) could be interpreted as nonconsummatory, and (3) occurred in the absence of the tone/solenoid cue, which presumably evokes a representation of water delivery; thus, licking during water-off phases could have been guided solely by antecedent stimuli (spout, hole in front wall, etc.) that only weakly predicted water delivery. Despite these apparent dissociations from water delivery, and characteristics which could be conducive to habit formation, licking during water-off phases was equally sensitive to devaluation in both early and late sessions, indicating that licking remained goal directed and not habitual.

Also unchanged over sessions were the number of licks and lick period, consistent with the minimal variation in licking, as part of the normal behavioral repertoire of rats (Hulse and Suter, 1968; Hernandez-Mesa et al., 1985) and consistent with the involvement of a brainstem oscillator (Wiesenfeld et al., 1977). Nevertheless, changes across sessions were observed. The rapid decline to stable asymptote in the latency to initiate licking upon resumption of audible tone/solenoid activation starting each water-on phase was indicative of learning the task's programmed timing of water availability and task learning. In conjunction, motor learning was also evidenced by reduced lick duration across sessions, hence more efficient licking. neurons.

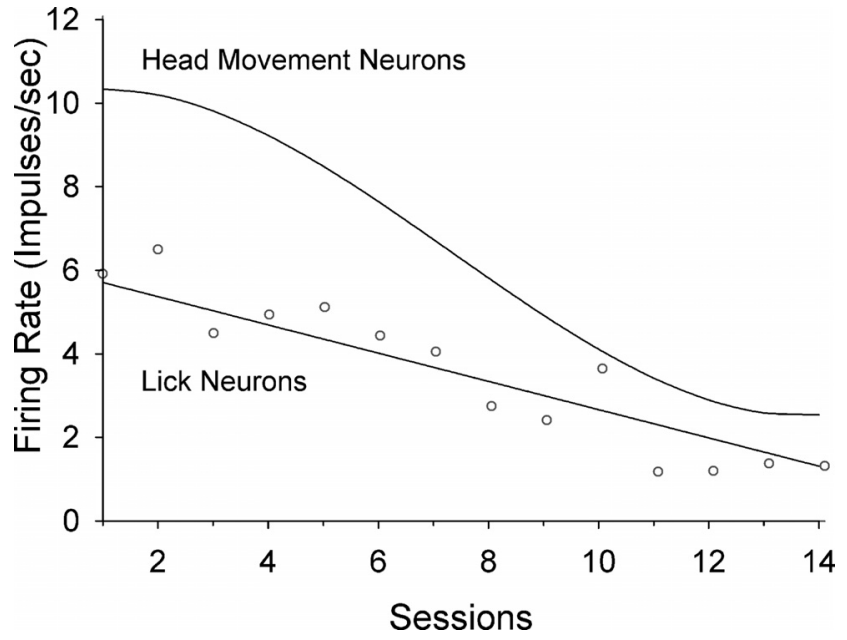

Figure 5. Model-predicted trajectories of average change in firing rate for lick neurons (bottom line) and for head movement neurons (top line) across 14 sessions of training. A linear decrease in firing rate was evident $(p<0.001)$ for lick neurons over 14 sessions in the lick task, with no habit formation. Mean firing rates (open circles) of matched sets for all lick neurons are plotted at each of the 14 sessions. Note that the linear trajectory was not a simple regression line of these mean values but rather a result of the three-level individual growth model on the neural data of matched sets of all neurons (see text). In contrast, a curvilinear decrease ( $p<$ 0.001 ) was present for head movement neurons over 14 sessions in the head movement task, with habit formation (Tang et al., 2007).

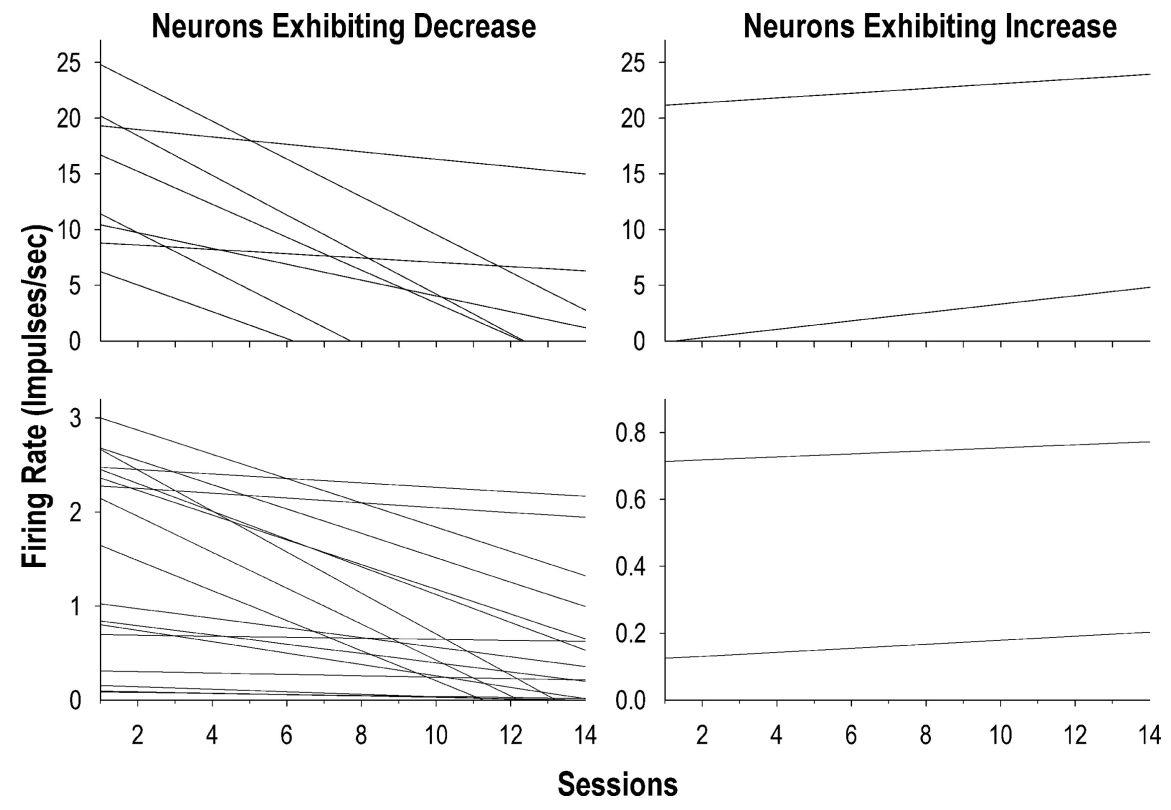

Figure 6. Model-predicted trajectories of average change in firing rate for individual lick neurons across sessions. The linear trend line for each neuron resulted from a two-level individual growth model on the matched sets of the neuron. Left, Twenty-five neurons exhibiting decreased firing rate over sessions; right, remaining four neurons exhibiting increased firing rate over sessions. For the purpose of better display, on either side, neurons were separately shown in the top or bottom with different scales of the $y$ axis, according to the initial firing rate and the rate of change in firing of individual

While substantial repetition of a movement and lengthy training may be required for skilled performance, they are not obligatory determinants or the only factors involved in motor habit formation. Characteristics of the movement, such as whether it is learned or not, may be additional essential factors in habit formation. Licking is arguably a natural, unconditioned behavior. Simply learning where and when to lick may require minimal striatal processing. Tang et al. (2007) observed im- 


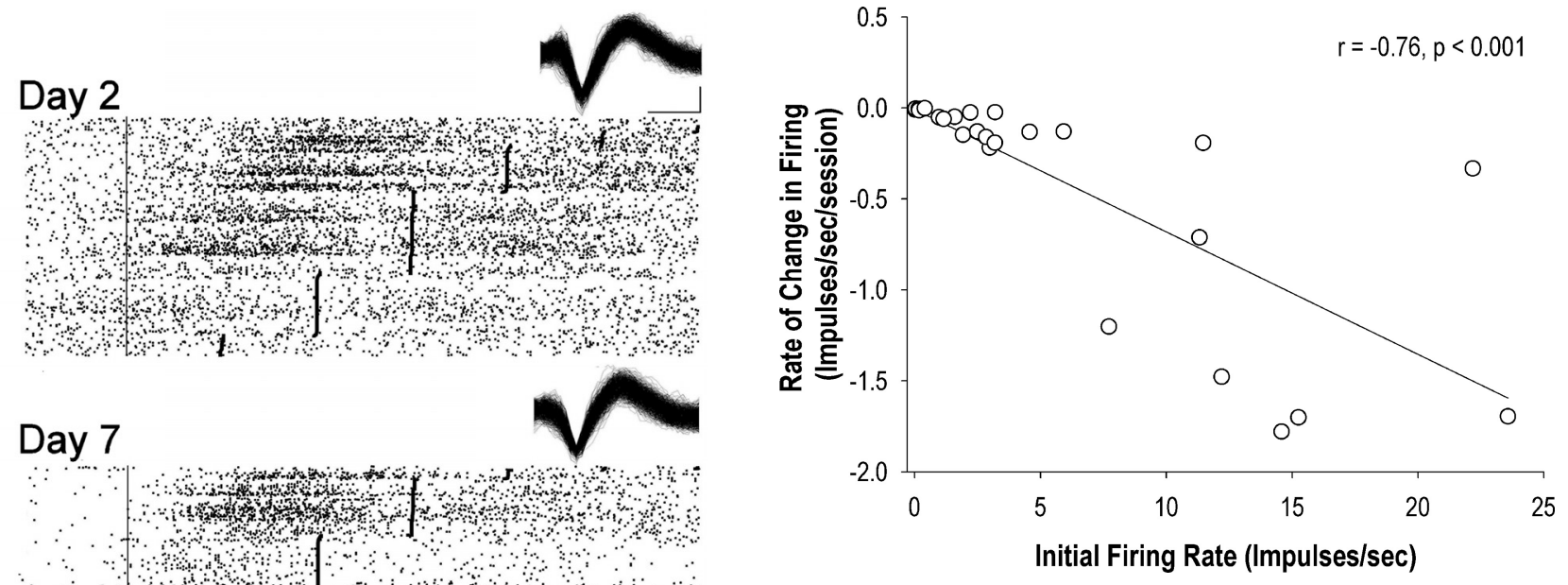

Figure 8. Negative correlation $(p<0.001)$ between the initial firing rate and the rate of decrease in firing rate of lick neurons $(n=25)$. In these neurons, higher initial firing rate was predictive of faster decline in firing over sessions in the lick task.

changes across sessions. In both studies, faster firing neurons declined more rapidly. Firing of both head movement neurons and lick neurons showed a range of correlations with movement parameters, which tended to be lost over sessions. Therefore, loss of behavioral correlation with striatal firing is likely to result from overtraining, regardless of whether a motor habit forms. Although FRs and behavioral correlations both decreased over sessions, there was no relationship between the behavioral correlations and mean FRs in the first session; i.e., neurons with higher behavioral correlations do not necessarily have higher FRs. Behavioral correlations in the first session also did not predict the rates of decrease in FR over sessions.

A key finding is the different trajectories of FR change between studies. Lick neurons exhibited a constant rate of decrease while head movement neurons (Fig. 5, top line) maintained firing at a high level in early sessions, decreased firing rapidly in middle sessions, and remained at low levels of firing in late sessions. The extensive training required for the instrumental head movement to become skilled and habitual may require a high level of striatal firing in early sessions and thus a delayed decrease in firing, putatively an electrophysiological indicator of impending acquisition of skill and/or habitual behavior. In contrast, learning in the licking task (decreased reaction time to the tone/solenoid cue and decreased lick duration) occurred primarily between the first and third sessions, presumably due to less intensive motor learning, corresponding to a reduced latency for striatal firing to decrease across sessions, and lack of habit formation.

Alternatives to this proposition are that habits can form over few days of training (Adams, 1982; Dickinson et al., 1983), and that a number of other factors may be critical for habit formation, such as conditioning (Pavlovian or instrumental) and behavior (appetitive or consummatory), etc. Therefore, another possible explanation is that dorsolateral striatal firing changes reflect asymptotic task learning or motor learning within the task over days and weeks and may be critical for the formation of a motor habit if a habit is formed. Depending on the movement and on environmental contingencies under which the movement takes place, a habit may or may not form. Under these terms, striatal firing predominantly decreases across days of task and motor learning, whether responses remain goal directed or become habitual. 


\section{References}

Adams CD (1982) Variations in the sensitivity of instrumental responding to reinforcer devaluation. Q J Exp Psychol 34:77-98.

Alexander GE, DeLong MR (1985) Microstimulation of the primate neostriatum. II. Somatotopic organization of striatal microexcitable zones and their relation to neuronal response properties. J Neurophysiol 53:14171430.

Alexander GE, DeLong MR, Strick PL (1986) Parallel organization of functionally segregated circuits linking basal ganglia and cortex. Annu Rev Neurosci 9:357-381.

Balleine B (1992) Instrumental performance following a shift in primary motivation depends on incentive learning. J Exp Psychol Anim Behav Process 18:236-250.

Balleine BW, Garner C, Gonzalez F, Dickinson A (1995) Motivational control of heterogenous instrumental chains. J Exp Psychol Anim Behav Process 21:203-217.

Blomeley CP, Kehoe LA, Bracci E (2009) Substance P mediates excitatory interactions between striatal projection neurons. J Neurosci 29:49534963.

Calabresi P, Saiardi A, Pisani A, Baik JH, Centonze D, Mercuri NB, Bernardi $\mathrm{G}$, Borrelli E (1997) Abnormal synaptic plasticity in the striatum of mice lacking dopamine D2 receptors. J Neurosci 17:4536-4544.

Carelli RM, West MO (1991) Representation of body by single neurons in the dorsolateral striatum of the awake, unrestrained rat. J Comp Neurol 309:231-249.

Carelli RM, Wolske M, West MO (1997) Loss of lever press-related firing of rat striatal forelimb neurons after repeated sessions in a lever pressing task. J Neurosci 17:1804-1814.

Cho J, West MO (1997) Distributions of single neurons related to body parts in the lateral striatum of the rat. Brain Res 756:241-246.

Cooke JD (1980) The organization of simple, skilled movements. In: Tutorials in motor behavior (Stelmach GE, Requin J, eds), pp 199-212. Amsterdam: North-Holland.

Crutcher MD, DeLong MR (1984a) Single cell studies of the primate putamen. I. Functional organization. Exp Brain Res 53:233-243.

Crutcher MD, DeLong MR (1984b) Single cell studies in the primate putamen II. Relations to direction of movement and pattern of muscular activity. Exp Brain Res 53:244-258.

Dickinson A (1985) Actions and habits: the development of behavioural autonomy. Phil Trans R Soc Lond B 308:67-78.

Dickinson A, Nicholas DJ, Adams CD (1983) The effect of the instrumental training contingency on susceptibility to reinforcer devaluation. Q J Exp Psychol 35B:35-51.

Ebrahimi A, Pochet R, Roger M (1992) Topographical organization of the projections from physiologically identified areas of the motor cortex to the striatum in the rat. Neurosci Res 14:39-60.

Faure A, Haberland U, Condé F, El Massioui N (2005) Lesion to the nigrostriatal dopamine system disrupts stimulus-response habit formation. J Neurosci 25:2771-2780.

Filion M, Tremblay L, Bédard PJ (1988) Abnormal influences of passive limb movement on the activity of globus pallidus neurons in parkinsonian monkeys. Brain Res 444:165-176.

Flaherty AW, Graybiel AM (1993) Two input systems for body representations in the primate striatal matrix: experimental evidence in the squirrel monkey. J Neurosci 13:1120-1137.

Flaherty AW, Graybiel AM (1994) Input-output organization of the sensorimotor striatum in the squirrel monkey. J Neurosci 14:599-610.

Galarce EM, Crombag HS, Holland PC (2007) Reinforcer-specificity of appetitive and consummatory behavior of rats after Pavlovian conditioning with food reinforcers. Physiol Behav 91:95-105.

Graybiel AM (2000) The basal ganglia. Curr Biol 10:R509-R511.

Hernandez-Mesa N, Mamedov Z, Bures J (1985) Operant control of the pattern of licking in rats. Exp Brain Res 58:117-124.

Holland PC (1979) Differential effects of omission contingencies on various components of Pavlovian appetitive conditioned responding in rats. J Exp Psychol Anim Behav Process 5:178-193.

Holland PC, Rescorla RA (1975) The effect of two ways of devaluing the unconditioned stimulus after first- and second-order appetitive conditioning. J Exp Psychol Anim Behav Process 1:355-363.

Holland PC, Straub JJ (1979) Differential effects of two ways of devaluing the unconditioned stimulus after Pavlovian appetitive conditioning. J Exp Psychol Anim Behav Process 5:65-78.
Hulse SH, Suter S (1968) One-drop licking in rats. J Comp Physiol Psychol $66: 536-539$.

Keele SW (1968) Movement control in skilled motor performance. Psychol Bull 70:387-403.

Kerfoot EC, Agarwal I, Lee HJ, Holland PC (2007) Control of appetitive and aversive taste-reactivity responses by an auditory conditioned stimulus in a devaluation task: a FOS and behavioral analysis. Learn Mem 14:581-589.

Kimura M, Kato M, Shimazaki H (1990) Physiological properties of projection neurons in the monkey striatum to the globus pallidus. Exp Brain Res 82:672-676.

Kincaid AE, Wilson CJ (1996) Corticostriatal innervation of the patch and matrix in the rat neostriatum. J Comp Neurol 374:578-592.

Kleim JA, Barbay S, Nudo RJ (1998) Functional reorganization of the rat motor cortex following motor skill learning. J Neurophysiol 80:33213325.

Kosobud AE, Harris GC, Chapin JK (1994) Behavioral associations of neuronal activity in the ventral tegmental area of the rat. J Neurosci 14:7117-7129.

Künzle H (1975) Bilateral projections from precentral motor cortex to the putamen and other parts of the basal ganglia. An autoradiographic study in Macaca fascicularis. Brain Res 88:195-209.

Künzle H (1977) Projections from the primary somatosensory cortex to basal ganglia and thalamus in the monkey Exp Brain Res 30:481-492.

Liles SL (1979) Topographic organization of neurons related to arm movement in the putamen. In: Advances in neurology (Chase TN, Wexler NS, Barbeau A, eds), pp 156-162. New York: Raven.

Liles SL, Updyke BV (1985) Projection of the digit and wrist area of precentral gyrus to the putamen: relation between topography and physiological properties of neurons in the putamen. Brain Res 339:245-255.

Lovinger DM, Tyler EC, Merritt A (1993) Short- and long-term synaptic depression in rat neostriatum. J Neurophysiol 70:1937-1949.

Marsden CD (1982) The mysterious motor function of the basal ganglia: the Robert Wartenberg Lecture. Neurol 32:514-539.

McFarland NR, Haber SN (2000) Convergent inputs from thalamic motor nuclei and frontal cortical areas to the dorsal striatum in the primate. J Neurosci 20:3798-3813.

McGeorge AJ, Faull RL (1989) The organization of the projection from the cerebral cortex to the striatum in the rat. Neuroscience 29:503-537.

Meyer DE, Abrams RA, Kornblum S, Wright CE, Smith JE (1988) Optimality in human motor performance: ideal control of rapid aimed movements. Psychol Rev 95:340-370.

Mishkin M, Petri H (1984) Memories and habits: some implications for the analysis of learning and retention. In: Neuropsychology of memory (Butters N, Squire LR, eds). New York: Guilford.

Mittler T, Cho J, Peoples LL, West MO (1994) Representation of the body in the lateral striatum of the freely moving rat: single neurons related to licking. Exp Brain Res 98:163-167.

Nudo RJ, Milliken GW, Jenkins WM, Merzenich MM (1996) Usedependent alterations of movement representations in primary motor cortex of adult squirrel monkeys. J Neurosci 16:785-807.

Parent A, Hazrati LN (1993) Anatomical aspects of information processing in primate basal ganglia. Trends Neurosci 16:111-116.

Pasupathy A, Miller EK (2005) Different time courses of learning-related activity in the prefrontal cortex and striatum. Nature 433:873-876.

Paxinos G, Watson C (2005) The rat brain in stereotaxic coordinates, Ed 5. San Diego: Elsevier Academic.

Pederson CL, Wolske M, Peoples LL, West MO (1997) Firing rate dependent effect of cocaine on single neurons of the rat lateral striatum. Brain Res 760:261-265.

Peoples LL, Gee F, Bibi R, West MO (1998) Phasic firing time locked to cocaine self-infusion and locomotion: dissociable firing patterns of single nucleus accumbens neurons in the rat. J Neurosci 18:7588-7598.

Raz A, Frechter-Mazar V, Feingold A, Abeles M, Vaadia E, Bergman H (2001) Activity of pallidal and striatal tonically active neurons is correlated in mptp-treated monkeys but not in normal monkeys. J Neurosci 21:RC128.

Rothblat DS, Schneider JS (1995) Alterations in pallidal neuronal responses to peripheral sensory and striatal stimulation in symptomatic and recovered parkinsonian cats. Brain Res 705:1-14.

Selemon LD, Goldman-Rakic PS (1985) Longitudinal topography and in- 
terdigitation of corticostriatal projections in the rhesus monkey. J Neurosci 5:776-794.

Sheridan MR, Flowers KA, Hurrell J (1987) Programming and execution of movement in Parkinson's disease. Brain 110:1247-1271.

Singer JD, Willett JB (2003) Applied longitudinal data analysis: modeling change and event occurrence. Oxford: Oxford UP.

Tang C, Mittler T, Duke DC, Pawlak AP, Zhu Y, Prokopenko VF, West MO (2005) Striatal neurons related to licking: dose- and rate-dependent effects of cocaine on firing rate concurrent with licking stereotypy in rats. Soc Neurosci Abstr 31:453.14.

Tang C, Pawlak AP, Prokopenko V, West MO (2007) Changes in activity of the striatum during formation of a motor habit. Eur J Neurosci 25:1212-1227.

Tang C, Mittler T, Duke DC, Zhu Y, Pawlak AP, West MO (2008) Dose- and rate-dependent effects of cocaine on striatal firing related to licking. J Pharmacol Exp Ther 324:701-713.
Tremblay L, Filion M, Bédard PJ (1989) Responses of pallidal neurons to striatal stimulation in monkeys with MPTP-induced parkinsonism. Brain Res 498:17-33.

West MO, Carelli RM, Pomerantz M, Cohen SM, Gardner JP, Chapin JK, Woodward DJ (1990) A region in the dorsolateral striatum of the rat exhibiting single-unit correlations with specific locomotor limb movements. J Neurophysiol 64:1233-1246.

Wickens JR, Mckenzie D, Costanzo E, Arbuthnott GW (1998) Effects of potassium channel blockers on synaptic plasticity in the corticostriatal pathway. Neuropharmacology 37:523-533.

Wiesenfeld Z, Halpern BP, Tapper DN (1977) Licking behavior: evidence of hypoglossal oscillator. Science 196:1122-1124.

Wise SP, Murray EA, Gerfen CR (1996) The frontal cortex-basal ganglia system in primates. Crit Rev Neurobiol 10:317-356.

Yin HH, Knowlton BJ, Balleine BW (2004) Lesions of the dorsolateral striatum abolish habits and preserve goal expectancy. Eur J Neurosci 19:181-189. 